\title{
ON $\mathfrak{\xi}$-SUBNORMAL SUBGROUPS AND FRATTINI-LIKE SUBGROUPS OF A FINITE GROUP
}

\author{
by A. BALLESTER-BOLINCHES and M. D. PÉREZ-RAMOS
}

(Received 3 November, 1992)

1. Introduction. Throughout the paper we consider only finite groups.

J. C. Beidleman and $\mathrm{H}$. Smith [3] have proposed the following question: "If $G$ is a group and $H$ a subnormal subgroup of $G$ containing $\Phi(G)$, the Frattini subgroup of $G$, such that $H / \Phi(G)$ is supersoluble, is $H$ necessarily supersoluble? "In this paper, we give not only an affirmative answer to this question but also we see that the above result still holds if supersoluble is replaced by any saturated formation containing the class of all nilpotent groups.

On the other hand, Doerk and the authors [2] introduce and study a new embedding property of a subgroup in a soluble group which is an extension of the classical normality, namely $\mathfrak{r}$-normality, where $\mathfrak{x}$ is a saturated formation of soluble groups. We prove here that if $G$ is a soluble group and $H$ is an $\tilde{r}$-normal subgroup of $G$ containing $\Phi(G)$ such that $H / \Phi(G)$ belongs to $\mathfrak{i}$ then $H$ belongs to $\mathfrak{s}$. Moreover, if $\mathfrak{s}$ is a subgroup-closed saturated formation, then the result is also true if $\mathfrak{\aleph}$-normal is replaced by $\mathfrak{\wp}$-subnormal.

Finally, we give some results related to Beidleman and Smith's question concerning the relationship between certain Frattini-like subgroups of a group $G$ and the supersolubility or nilpotency of some subnormal subgroups of $G$.

2. Preliminaries. The reader is assumed to be familiar with the theory of saturated formations of finite groups. We shall adhere to the notation used in [5] and we refer the reader to that book for the basic notation, terminology and results.

For the sake of completeness, we give some definitions and results used in proving our Theorems.

Recall that if $\tilde{\xi}$ is a saturated formation and $G$ is a group, a maximal subgroup $M$ of a group $G$ is said to be $\uparrow$-normal in $G$ if the primitive group $G / \operatorname{Core}_{G}(M) \in \Gamma$ and

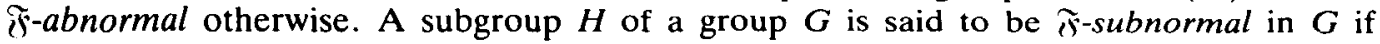
either $H=G$ or there exists a chain

$$
H=H_{n} \leq H_{n-1} \leq \ldots \leq H_{0}=G
$$

such that $H_{i+1}$ is a maximal $\mathfrak{i}$-normal subgroup of $H_{i}$, for every $i=0, \ldots, n-1$.

Definition 2.1 ([2]). Denote by $\mathfrak{f}$ the smallest local definition of the saturated formation $\mathfrak{i}$ (cf. [5; IV, 3.9]). A subgroup $H$ of a group $G$ is said to be $\mathfrak{F}$-normal in $G$ if $H /$ Core $_{G}(H) \in \mathfrak{f}(p)$ for every prime $p$ dividing $|G: H|$, the index of $H$ in $G$.

It is clear that if $\mathfrak{\uparrow}=\Downarrow$, the saturated formation of all nilpotent groups, then $\mathfrak{M}$-normality coincides with the classical normality. Moreover, a maximal subgroup of a group is $\mathfrak{\$}$-normal in the sense of 2.1 if and only if it is $\mathbb{\$}$-normal in the classical sense and a subgroup $H$ of a group $G$ is $\mathfrak{乛}$-subnormal in $G$ if and only if $H$ can be joined to $G$ by means of a chain of $\mathfrak{\aleph}$-normal subgroups (cf. [2]).

It is not difficult to prove that if $N \unlhd G$ and $H$ is $\widetilde{S}$-normal (respectively $\mathfrak{\wp}$-subnormal) in $G$, then $H N / N$ is $\mathfrak{\wp}$-normal (respectively $\mathfrak{r}$-subnormal) in $G / N$.

Let $K$ be a field of characteristic $p$ ( $p$ a prime number) and let $G$ be a group.

Glasgow Math. J. 36 (1994) 241-247. 
Consider $K$ as trivial $K G$-module and denote by $P_{K}$ the indecomposable projective $K G$-module with $K$ in the head, i.e. $P_{K} / P_{K} J \cong K$, where $J$ is the Jacobson radical of $K G$ (we consider only finite modules). $A_{K}(G)$ denotes the kernel of a projective cover $P_{1} \rightarrow P_{K} J ; A_{K}(G)$ is uniquely determined up to isomorphism.

Theorem 2.2 (W. Gaschütz, see [8]). Let $K=\mathrm{GF}(p)$, the finite field of $p$ elements, and $A=A_{K}(G)$. Then there exists a Frattini extension $A \mapsto G^{*} \rightarrow G$, i.e. with $A \leq \Phi\left(G^{*}\right)$. Any other Frattini extension of $G$ by a $K G$-module is an epimorphic image over $G$ of $G^{*}$.

$A_{K}(G)$ is called the Frattini module of $G$ with respect to $K$. It is known that $A_{K}(G) \neq 0$ if $p$ divides the order of $G$; for a $p^{\prime}$-group $G, A_{K}(G)=0$. Moreover, $A_{K}(G)$ is in the first block of the group algebra $K G$. So, by a well known result of Brauer, $O_{p^{\prime}}(G) \leq C_{G}\left(A_{K}(G)\right)$.

Let $\mathfrak{i}$ be a saturated formation. For any group $G$, denote by $L_{\pi}(G)$ the intersection of all $\tilde{r}$-abnormal maximal subgroups of $G$, with the usual provision that the subgroup concerned equals $G$ if no such maximals exist (cf. $[1,6]$ ). When $\mathfrak{i}=\mathbb{l}$, the class of all supersoluble groups, we denote $L_{\mathrm{II}}(G)$ simply by $L(G)$.

Let $G$ be a group and $\pi$ a set of primes. We consider the following families of maximal subgroups of $G$ :

$M_{1}(G)=\left\{M \mid M\right.$ is a maximal subgroup of $G$ and $\left.|G: M|_{\pi}=1\right\}$, where $|G: M|_{\pi}$ denotes the $\pi$-part of the index of $M$ in $G$.

$\mathcal{M}_{2}(G)=\left\{M \in \mathcal{M}_{1}(G)|| G: M \mid\right.$ is a composite number $\}$.

Denote by $\Phi_{\pi}(G)=\bigcap\left\{M \mid M \in \mathcal{M}_{1}(G)\right\}$ and by $S_{\pi}(G)=\bigcap\left\{M \mid M \in \mathcal{M}_{2}(G)\right\}$. As usual, $\Phi_{\pi}(G)=G$ if $\mathcal{M}_{1}(G)$ is empty and $S_{\pi}(G)=G$ if $\mathcal{M}_{2}(G)$ is empty. Clearly, $\Phi_{\pi}(G)$ and $S_{\pi}(G)$ are characteristic subgroups of $G$ such that $\Phi(G) \cup O_{\pi}(G) \subseteq \Phi_{\pi}(G) \leq S_{\pi}(G)$, where $O_{\pi}(G)$ is the maximal normal $\pi$-subgroup of $G$. These Frattini-like subgroups were introduced by Bhattacharya and Mukherjee in [4].

The next result is used frequently in induction arguments. Its proof is simple.

Proposition 2.3. Denote by $T(G)$ any one of the Frattini-like subgroups defined above. For every $N \unlhd G$, we have that $T(G) N / N \leq T(G / N)$ and if $N \leq T(G)$, then $T(G) N / N=T(G / N)$.

3. $\mathfrak{s}$-normal and $\mathfrak{s}$-subnormal subgroups. In the sequel, $\mathfrak{s}$ will denote a saturated formation. By $[5 ; \mathrm{IV}, 3.8]$, there exists a unique formation function, $F$ say, defining $\mathfrak{F}$ which is integrated and full. We denote by $f$ the smallest local definition of $\mathfrak{F}$ and by $\pi$ the characteristic of $\mathfrak{s}$, that is, $\pi=\{p \in \mathbb{P} \mid(F(p)$ is non-empty $\}$.

THEOREM 3.1. Let $G$ be a group and $H$ a subnormal subgroup of $G$ containing $O_{\pi}(\Phi(G))$ such that $H / O_{\pi}(\Phi(G))$ belongs to $\mathfrak{F}$. Then $H$ belongs to $\mathfrak{F}$.

Proof. (M. J. Tomkinson). By [5; IV, 4.3], $\mathfrak{\&}$ is contained in $\mathfrak{F}_{\pi}$, the class of all $\pi$-groups. Without loss of generality we can assume that $\Phi(G)$ is a $\pi$-group. Thus, $H$ is a $\pi$-group and $H / \Phi(G)$ is an $\uparrow$-group. Let $p$ be a prime in $\pi$. Since $H$ is a subnormal subgroup of $G$ and $O_{p^{\prime} p}(G / \Phi(G))=O_{p^{\prime} p}(G) / \Phi(G)$, we have that $O_{p \cdot p}(H / \Phi(G))=$ $O_{p^{\prime} p}(H) / \Phi(G)$. Now, $H / \Phi(G)$ is an $\overleftarrow{\$}$-group. This means that $(H / \Phi(G)) / O_{p^{\prime} p}(H / \Phi(G)) \in F(p)$ and so $H / O_{p^{\prime} p}(H) \in F(p)$. Therefore $H$ is an $\mathfrak{s}$-group.

REMARKS. If $\mathfrak{f}$ contains $\mathfrak{U}$, the class of all nilpotent groups, then $\pi=\mathbb{P}$, the set of all prime numbers. So, what Theorem 3.1 says in this case is the following: "If $G$ is a group 
and $H$ a subnormal subgroup of $G$ such that $H / \Phi(G) \in \mathfrak{F}$, then $H \in \mathfrak{F}$ ". In particular, when $\mathfrak{i}$ is the saturated formation of all supersoluble groups, we have an affirmative answer to the question of Beidleman and Smith.

The above result is not true if $\mathfrak{s}$ is not of full characteristic. Let $\mathfrak{s}=\Xi_{p}$ be the saturated formation of all $p$-groups, $p$ a prime number. Take a prime $q \neq p$ and let $G=C_{p^{2}} \times C_{q^{2}}$ be the cyclic group of order $p^{2} q^{2}$. If $H=C_{p^{2}} \Phi(G)$, then $H \unlhd G$ and $H / \Phi(G) \in \mathfrak{\Uparrow}$ but $H \notin \mathfrak{r}$.

The following result was communicated to the authors by M. J. Tomkinson.

COROllary 3.2. Assume that î is closed under taking subnormal subgroups. Let $G$ be a group and $H$ a subnormal subgroup of $G$ containing $O_{\pi}\left(L_{\pi}(G)\right)$ such that $H / O_{\pi}\left(L_{\Re}(G)\right) \in \mathfrak{T}$. Then $H \in \mathfrak{r}$.

Proof. By [5; IV, 3.16], $F(p)$ is closed under taking subnormal subgroups for every $p \in \pi$. Assume that the result is not true and take $G$ a counterexample of minimal order. A routine argument shows that $G$ has a unique minimal normal subgroup $N$ such that $N \leq O_{\pi}\left(L_{\pi}(G)\right)$ and $H / N \in \widetilde{\uparrow}$. In particular, $H$ is a $\pi$-group. If $N \leq \Phi(G)$ then $H \in \Re$ by (3.1), a contradiction. Therefore there exists a maximal subgroup $M$ of $G$ such that $G=M N$. Now, $M$ is $\tilde{r}$-normal in $G$ because $N \leq L_{\pi}(G)$. This means that $G / C_{G}(N) \epsilon$ $F(p)$ for each prime $p$ dividing $|N|$. Hence $H / C_{H}(N) \in F(p)$ for every prime $p$ dividing $|N|$ and so each chief factor of $H$ below $N$ is $\mathfrak{F}$-central in $H$. Applying [5; IV, 3.5(c)], we have that $H \in \mathfrak{N}$, a contradiction.

Corollary 3.3. Assume that is is closed under taking subnormal subgroups. Let $G$ be

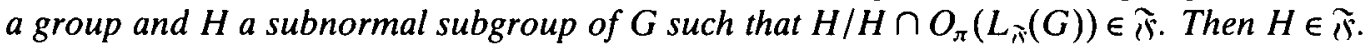

Taking $H=O_{\pi}\left(L_{\pi}(G)\right)$ in the above Corollary, we obtain statement (1) of the Theorem of Feng and Zhang [6].

The assumption on $\mathfrak{f}$ in the above result is necessary. To see that this is so, it is enough to consider a saturated formation $\mathfrak{\nwarrow}$ of full characteristic which is not closed under taking subnormal subgroups (see example 3.8 below). In this case, there exists a group $G \in \mathfrak{i}$ with a subnormal subgroup $H$ such that $H \notin \Re$. Thus $L_{\pi}(G)=G$ and then $H / H \cap O_{\pi}\left(L_{\pi}(G)\right)=H / H \in \mathfrak{r}$.

The following result is a particular case of Corollary 3.3 and can be considered as a generalization of the Corollary of [3] in the universe of all finite groups. It will be used in Section 4.

Corollary 3.4. Let $G$ be a group and assume that $H$ is a subnormal subgroup of $G$ such that $H / H \cap L(G)$ is supersoluble. Then $H$ is supersoluble.

Next we see how the above results can be extended to $\mathfrak{\wp}$-normal and $\mathfrak{f}$-subnormal subgroups.

THEOREM 3.5. Let $G$ be a soluble group and $H$ an $\mathfrak{i}$-normal subgroup of $G$. Assume there exists a normal subgroup $M$ of $G$ such that $M \leq H \cap \Phi(G)$ and $H / M \in \mathfrak{i}$. Then $H \in \mathfrak{r}$.

Proof. First of all, notice that $G$ is a $\pi$-group where $\pi=\operatorname{char} \approx$ because $H$ is $\Re$-normal in $G$ and $H / M$ is a $\pi$-group. 
Assume that the result is not true and let $G$ be a counterexample of minimal order. Let $N$ be a minimal normal subgroup of $G$. The group $G / N$ and the pair $(H N / N, M N / N)$ satisfy the hypotheses of the theorem. So $H N / N \in \mathfrak{F}$ by our choice of $G$. If $W$ is another minimal normal subgroup of $G$ then again $H W / W \in \mathfrak{\uparrow}$. Hence $H \in \mathfrak{\uparrow}$, a contradiction. Consequently, $N=\operatorname{Soc}(G)$ is the unique minimal normal subgroup of $G$. Since $M \neq 1$, it is clear that $N \leq M \leq H \cap \Phi(G), N$ is abelian and $H / N \in \mathfrak{r}$. Denote by $p$ the prime dividing $|N|$ and let $K$ be the finite field of $p$ elements. Consider the Frattini extension of $G^{*}=G / N: N \mapsto G \rightarrow G^{*}$. By Theorem $2.2 N$, regarded as a $K G^{*}$-module by conjugation, is a quotient module of $A=A_{K}\left(G^{*}\right)$, the Frattini module of $G^{*}$ with respect to $K$. Since $O_{p^{\prime}}\left(G^{*}\right) \leq C_{G^{*}}(A)$, we have that $O_{p^{\prime}}\left(G^{*}\right) \leq C_{G^{*}}(N)$. Let $T=O_{p^{\prime}}(G \bmod N)$. By the Schur-Zassenhaus Theorem, $T=Q N$ where $Q$ is a Hall $p^{\prime}$-subgroup of $T$. Now, $Q$ is a normal $p^{\prime}$-subgroup of the normal subgroup $T$ of $G$ because $Q$ centralizes $N$. Hence $Q \leq O_{p},(G)=1$. Thus $O_{p^{\prime}}\left(G^{*}\right)=1$. Assume that $p$ does not divide $|G: H|$. Then $H^{*}=H / N$ contains a Sylow $p$-subgroup of $G^{*}$. In particular, $O_{p}\left(G^{*}\right) \leq H^{*}$. But $F\left(G^{*}\right)=O_{p}\left(G^{*}\right)$. So $C_{G^{*}}\left(O_{p}\left(G^{*}\right)\right) \leq O_{p}\left(G^{*}\right)$ because $G^{*}$ is soluble. Now, $O_{p}\left(H^{*}\right)$ centralizes $O_{p}\left(G^{*}\right)$ and then $O_{p}\left(H^{*}\right)=1$. Therefore $O_{p}\left(H^{*}\right)=O_{p}{ }^{\prime}\left(H^{*}\right)$ and since

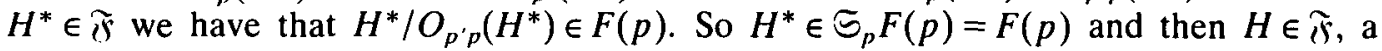
contradiction. Therefore $p$ divides $|G: H|$. Since $H$ is $\tilde{\xi}$-normal in $G$, we have that $H /$ Core $_{G}(H) \in \mathfrak{f}(p)$. In particular, the $\mathfrak{f}(p)$-residual, $H^{\mathrm{i}(p)}$, of $H$ is subnormal in $G$. Then $O_{p} \cdot\left(\left(H^{*}\right)^{i(p)} \leq O_{p^{\prime}}\left(G^{*}\right)=1\right.$. On the other hand, $\left(H^{*}\right)^{i(p)}$ is $p$-nilpotent because $H^{*} \in \mathfrak{i}$. Therefore $H^{*} \in \Xi_{p} \mathfrak{f}(p)=F(p)$ and then $H \in \Xi_{p} F(p)=F(p) \subseteq \mathfrak{F}$, the final contradiction.

EXAMPLE 3.6. Let $p$ be a prime number and consider $\mathfrak{F}=\Xi_{p} \cdot \Xi_{p}$ the saturated formation of soluble $p$-nilpotent groups. The smallest local definition $f$ of $\mathfrak{f}$ is given by $\mathfrak{f}(p)=1$ and $\mathfrak{f}(q)=\Xi_{p}, \Xi_{p}$ if $q \neq p$.

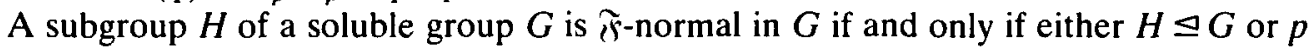
does not divide $|G: H|$ and $H /$ Core $_{G}(H) \in \Xi_{p}, \Xi_{p}$. Consequently, by Theorem 3.5 , if $H$ is a subgroup of a soluble group $G$ such that $H / \Phi(G)$ is $p$-nilpotent and either $H \unlhd G$ or $p$ does not divide $|G: H|$ then $H$ is likewise $p$-nilpotent.

THEOREM 3.7. Assume that $\mathfrak{i}$ is a subgroup-closed formation. Let $G$ be a soluble group and $H$ an $\mathfrak{\$}$-subnormal subgroup of $G$. Suppose there exists a normal subgroup $M$ of $G$ such that $M \leq H \cap \Phi(G)$ and $H / M \in \mathfrak{x}$. Then $H \in \mathfrak{r}$.

Proof. As in the above Theorem, it is not difficult to prove that $G$ is a $\pi$-group where $\pi=$ char $\mathfrak{F}$. Suppose the result is not true and consider $G$ a counterexample of minimal order. With similar arguments to those used in the above result, we have that $N=\operatorname{Soc}(G)$ is the unique minimal normal subgroup of $G, N \leq M \leq H \cap \Phi(G), N$ is abelian and $H / N \in \mathfrak{i s}$. Denote by $p$ the prime dividing $|N|$. We denote by stars the images in $G^{*}=G / N$. Again, as in the above Theorem, we have that $O_{p},\left(G^{*}\right)=1$. Moreover, if $O_{p}\left(G^{*}\right) \leq H^{*}$, then $O_{p},\left(H^{*}\right)=1$ and $H \in \mathfrak{F}$. Therefore we can assume that $H^{*}$ is a proper subgroup of $H^{*} O_{p}\left(G^{*}\right)$. By [7; Lemma 1.1], $H^{*}$ is an $\mathfrak{\kappa}$-subnormal subgroup of $H^{*} O_{p}\left(G^{*}\right)$. So there exists a chain

$$
H^{*}=H_{n} \leq H_{n-1} \leq \ldots \leq H_{0}=H^{*} O_{p}\left(G^{*}\right)
$$

such that $H_{i+1}$ is a maximal $\mathfrak{i}$-normal subgroup of $H_{i}$, for every $i=0, \ldots, n-1$. Take an index $i \in\{0, \ldots, n-1\}$. Since $H_{i+1}$ is a maximal $\mathfrak{\uparrow}$-normal subgroup of $H_{i}$, we have that the $F(p)$-residual, $\left(H_{i+1}\right)^{F(p)}$, of $H_{i+1}$ is a subnormal subgroup of $H_{i}$. Moreover, 
$\left(H_{i+1}\right)^{F(p)} \leq\left(H_{i}\right)^{F(p)}$ because $F(p)$ is subgroup-closed (see [5; IV , 3.16]). This implies that $\left(H^{*}\right)^{F(p)}$ is a subnormal subgroup of $H^{*} O_{p}\left(G^{*}\right)$ and $O_{p} \cdot\left(\left(H^{*}\right)^{F(p)}\right) \leq O_{p} \cdot\left(H^{*} O_{p}\left(G^{*}\right)\right)$. Hence $O_{p^{\prime}}\left(\left(H^{*}\right)^{F(p)}\right) \leq C_{G^{*}}\left(O_{p}\left(G^{*}\right)\right) \leq O_{p}\left(G^{*}\right)$ and so $O_{p} \cdot\left(\left(H^{*}\right)^{F(p)}\right)=1$. But $\left(H^{*}\right)^{F(p)}$ is $p$-nilpotent because $H^{*} \in \mathfrak{s}$. Consequently, $\left(H^{*}\right)^{F(p)}$ is a $p$-group and then $H \in \Xi_{p} F(p)=$ $F(p) \subseteq \Re$, a contradiction.

If $\mathfrak{i}$ is not a subgroup closed saturated formation the above Theorem fails as the next example shows.

EXAMPLE 3.8. Let $\mathfrak{i}$ be the saturated formation locally defined by the formation function $f$ given by the formula: $f(p)=(1)$ if $p \neq 2,3$ and $f(2)=f(3)=(G \mid G$ is soluble and the Carter subgroups of $G$ are 2-groups). It is easy to see that $\tilde{\delta}$ is not a subgroup-closed formation.

Let $A$ be the symmetric group of degree three. There exists an irreducible and faithful $A$-module $V$ over $G F(2)(\operatorname{dim} V=2)$. Let $P=H(\mathbb{V})$ be the Hartley group on $V=\{V, V\}$ (see [5; B, 12.11]). Regarding $A$ as an operator group for $P$, we have $P / \Phi(P)$ is $A$-isomorphic to $V \oplus V$ and $\Phi(P)$ is $A$-isomorphic to $V \otimes V$. Consider $G=[P] A$ the corresponding semidirect product and the subgroup $H=J B$, where $J=\Phi(P)$ and $B=O_{3}(A)$. Then $H / J \in \mathfrak{s}$. Since $V \otimes V$ is a faithful $A$-module, we have that $H^{f(2)}$ is not 2-nilpotent. Therefore $H$ is not an $\mathfrak{\wp}$-group. Now, $H$ is a normal subgroup of $J A$ and $J A$ is

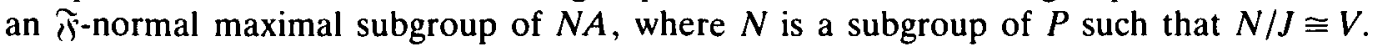
Moreover, $N A$ is an $\Re$-normal maximal subgroup of $G$. Therefore $H$ is an $\aleph^{\text {-subnormal }}$ subgroup of $G$.

4. Frattini-like subgroups. In this section, we consider the relationship between the Frattini-like subgroups $\Phi_{\pi}(G)$ and $S_{\pi}(G)$ of a group $G$ and the supersolubility or nilpotency of some subnormal subgroups of $G$.

Feng and Zhang [6] proved that $\Phi_{\pi}(G)=\Phi\left(G \bmod O_{\pi}(G)\right)$ and, if $S_{\pi}(G)$ is $\pi$-separable, then $S_{\pi}(G) / O_{\pi}(G)$ is supersoluble. As a consequence the commutator subgroup of $S_{\pi}(G) / O_{\pi}(G)$ is nilpotent. Our first result in this section shows that rather more than this is true.

THeOrem 4.1. Let $G$ be a group. If $S_{\pi}(G)$ is $\pi$-separable then $\left(G^{\prime} \cap S_{\pi}(G)\right) /\left(G^{\prime} \cap\right.$ $\left.O_{\pi}(G)\right)$ is a nilpotent group.

Proof. Again we argue by induction on the order of $G$. Since $O_{\pi}\left(G / O_{\pi}(G)\right)=1$ and $S_{\pi}\left(G / O_{\pi}(G)\right)=S_{\pi}(G) / O_{\pi}(G)$, we can assume that $O_{\pi}(G)=1$ and $H \neq 1$ where $H$ is the subgroup $G^{\prime} \cap S_{\pi}(G)$. Let $N$ be a minimal normal subgroup of $G$ contained in $H$ and suppose that $O_{\pi}(G / N)=1$. By induction hypothesis, $H / N$ is nilpotent. Since $S_{\pi}(G)$ is a supersoluble group and $O_{\pi}(G)=1$, we have that $N$ is an abelian $\pi^{\prime}$-group. On the other hand, $H$ is soluble. Therefore, by $[5 ; \mathrm{III}, 4.6] ; H$ has a conjuacy class of nilpotent projectors, the Carter subgroups of $H$. Let $C$ be a Carter subgroup of $H$. Then $H=N C$, $G=H N_{G}(C)$ and $G=N N_{G}(C)$. If $C$ were a normal subgroup of $G$ then $H$ would be nilpotent and we are done. Otherwise, let $M$ be a maximal subgroup of $G$ containing $N_{G}(C)$. Since $N$ is a $\pi^{\prime}$-group, $G=M N$ and $N \leq H$ it follows that $|G: M|_{\pi}=1$ and $|G: M|$ is a prime number. This implies that $N$ is a cyclic group of prime order and thus $G / C_{G}(N)$ is abelian. Consequently, $N \leq Z(H)$ and $H$ is nilpotent. Therefore we can assume that $O_{\pi}(G / N) \neq 1$ for every minimal normal subgroup $N$ of $G$ which is contained in $H$. Let $N$ 
be one of them and denote by $T$ the subgroup of $G$ such that $T / N=O_{\pi}(G / N) \cap G^{\prime} / N$. By the Schur-Zassenhaus Theorem, there exists a Hall $\pi$-subgroup $Q$ of $T$ such that $T=Q N$ and $G=N N_{G}(Q)$. Since $O_{\pi}(G)=1$, we have $N_{G}(Q)<G$. Let $M$ be a maximal subgroup of $G$ such that $N_{G}(Q) \leq M$. Since $N$ is a $\pi^{\prime}$-group, $G=M N$ and $N \leq H$ we have that $|G: M|_{\pi}=1$ and $|G: M|$ is a prime number. Thus $N$ is a cyclic group of prime order. Therefore each minimal normal subgroup of $G$ contained in $H$ is a cyclic group of prime order and $H \cap \Phi(G)=1$. Let $A$ be the product of all minimal normal subgroups of $G$ contained in $H$. By [9, Hilfssatz 4.4], there exists a subgroup $R$ of $G$ such that $G=A R$ and $A \cap R=1$. Clearly, $A \leq Z(H)$ and so $F(H) \cap R$, where $F(H)$ is the Fitting subgroup of $H$, is a normal subgroup of $G$. Now, $F(H) \cap R$ must be trivial because $A$ contains every minimal normal subgroup $N$ of $G$ such that $N \leq H$. Therefore $A=F(H)$ and $H \leq C_{H}(F(H)) \leq F(H)$ because $H$ is soluble. This means that $H$ is a nilpotent group.

By a result of Beidleman and Smith [3; Theorem 4], we have $G^{\prime \prime} \cap L(G) \leq \Phi(G)$ for all $G$. The corresponding result for $S_{\pi}(G)$ is the following:

THEOREM 4.2. Let $G$ be a group such that $S_{\pi}(G)$ is a $\pi$-separable group. Then $G^{\prime \prime} \cap S_{\pi}(G)$ is contained in $\Phi_{\pi}(G)$.

Proof. Let $G$ be a group such that $S_{\pi}(G)$ is a $\pi$-separable group and put $H=G^{\prime \prime} \cap S_{\pi}(G)$. In order to show $H \leq \Phi_{\pi}(G)$ we may asssume that $\Phi_{\pi}(G)=1$. Suppose, by contradiction, that $G$ is of minimal order subject to $\Phi_{\pi}(G)=1$ and $H \neq 1$. Since $\Phi_{\pi}(G)=1$, we have $O_{\pi}(G)=1$ and, by Theorem $4.1, H$ is a nilpotent normal subgroup of $G$. Moreover, $\Phi(G)=1$. Therefore $\Phi(H)=1$ and $H$ is abelian. Let $N$ be a minimal normal subgroup of $G$ contained in $H$. Since $\Phi_{\pi}(G)=1$ and $N \leq S_{\pi}(G)$, we can find a maximal subgroup $M$ of $G$ such that $|G: M|$ is a prime number, $|G: M|_{\pi}=1$ and $G=M N$. Thus $N$ is a cyclic group of prime order. Consider the primitive group $G^{*}=G / M_{G}$. If $M_{G} \neq 1$, then $G^{\prime \prime} M_{G} / M_{G} \cap S_{\pi}\left(G / M_{G}\right) \leq \Phi_{\pi}\left(G^{*}\right)$ by minimality of $G$. But $N^{*}=N M_{G} / M_{G}$ is the unique minimal normal subgroup of $G^{*}$ and $N^{*}$ is a $\pi^{\prime}$-group. Hence $O_{\pi}\left(G^{*}\right)=1$ and $\Phi_{\pi}\left(G^{*}\right)=1$. This implies that $H \leq M_{G}$ and $G=M$, a contradiction. Consequently, $M_{G}=1$ and $G$ is a primitive group. But then $F(G)=\operatorname{Soc}(G)$ is the unique minimal normal subgroup of $G$. Thus $N=F(G)$ and $G / N$ is a cyclic group. In particular, $G^{\prime \prime}=1$ and $H=1$, the required contradiction.

It should be noted that our Theorem 4.2 is not a corollary of Theorem 4 of [3] because there exist $\pi$-separable groups $G$ such that $L\left(G \bmod \Phi_{\pi}(G)\right) \neq S_{\pi}(G)$.

Our final theorem establishes the supersolubility of $H / O_{\pi}(G)$ if $H$ is a subnormal subgroup of $G$ such that $H / S_{\pi}(G)$ is supersoluble.

THEOREM 4.3. Let $G$ be a group such that $S_{\pi}(G)$ is a $\pi$-separable group. Assume that $H$ is a subnormal subgroup of $G$ containing $S_{\pi}(G)$. Then $H / O_{\pi}(G)$ is supersoluble if and only if $H / S_{\pi}(G)$ is supersoluble.

Proof. Since $O_{\pi}(G) \leq S_{\pi}(G)$, the necessity is obvious. Let $H / S_{\pi}(G)$ be supersoluble. We prove that $H / O_{\pi}(G)$ is supersoluble by induction on $|G|$. Clearly we can suppose that $O_{\pi}(G)=1$ and $S_{\pi}(G) \neq 1$. Let $N$ be a minimal normal subgroup of $G$ such that $N \leq S_{\pi}(G)$. Since $S_{\pi}(G)$ is supersoluble, we have that $N$ is an abelian $\pi^{\prime}$-group. If $O_{\pi}(G / N)=1$, then $H / N$ is supersoluble by induction. But $N \leq L(G)$. By Corollary 3.4, $H$ is supersoluble and we are done. So we may assume that $O_{\pi}(G / N) \neq 1$ for each 
minimal normal subgroup $N$ of $G$ contained in $S_{\pi}(G)$. Arguing as in the proof of Theorem 4.1, $N$ is a cyclic group of prime power order and moreover $N \cap \Phi(G)=1$. In particular, $\Phi(G)=1$. Moreover, $F\left(S_{\pi}(G)\right)$ is the product of all minimal normal subgroups of $G$ which are contained in $S_{n}(G)$. Since $H$ is a subnormal subgroup of $G$, it follows that $\Phi(H)=1$. Assume that $H$ is not supersoluble. Clearly $H$ is soluble. By [5; IV, 5.7] there exists a maximal subgroup $M$ of $H$ such that $H=M F(H)$ and $H /$ Core $_{H}(M)$ is not supersoluble. Since $\Phi(H)=1, F(H)$ is a direct product of minimal normal subgroups of $H$. Consequently, there exists a minimal normal subgroup $P$ of $H$ such that $H=M P$. On the other hand, $P$ is contained in $S_{\pi}(G)$ because $H / S_{\pi}(G)$ is supersoluble and $P$ is not a cyclic group. Thus $P \leq F\left(S_{\pi}(G)\right)$. But the index of $M$ in $H$ is not a prime number because $H / M_{H}$ is not supersoluble. This implies that $F\left(S_{n}(G)\right) \leq M$ and so $H=M$, a contradiction. Therefore $H$ is supersoluble and the theorem is proved.

ACKNOWLEDGMENT. We are grateful to Dr. M. J. Tomkinson for some useful remarks which have helped to improve this paper. This research was supported by DGICYTproyecto no. PB90-0414-C03-01.

\section{REFERENCES}

1. A. Ballester-Bolinches, Maximal subgroups and formations. J. Pure Appl. Algebra 61 (1989), 223-232.

2. A. Ballester-Bolinches, K. Doerk and M. D. Pérez-Ramos, On $\mathfrak{i}$-normal subgroups of finite soluble groups, preprint. 95-98.

3. J. C. Beidleman and H. Smith, On Frattini-like subgroups, Glasgow Math. J. 35 (1993),

4. P. Bhattacharya and N. P. Mukherjee, On the intersection of a class of maximal subgroups of a finite group II, J. Pure Appl. Algebra 42 (1986), 117-124.

5. K. Doerk and T. O. Hawkes, Finite soluble groups (De Gruyter, Berlin-New York, 1992).

6. Y. Feng and B. Zhang, Frattini subgroups relative to formation functions, J. Pure Appl. Algebra 64 (1990), 145-148.

7. P. Förster, On finite groups all of whose subgroups are $\mathfrak{乛}$-subnormal or $\widetilde{\Upsilon}$-subabnormal, $J$. Algebra 103 (1986), 285-293.

8. R. L. Griess and P. Schmid, The Frattini module, Arch. Math. 30 (1978), 256-266.

9. B. Huppert, Endliche Gruppen I (Springer-Verlag, Berlin, 1967).

Departament d'Algebra

UNIVERSITAT DE VALENCIA

C/Dr. Moliner 50

46100 BurJassot (VAlencia)

SpaIN 